\title{
Prescribers' Use of Electronic Prescribing Inquiry Tools in Puerto Rico
}

\author{
José J Hernández-Muñoz ${ }^{1^{*}}$, Caroline M Mejías De Jesús ${ }^{2}$, Carlos R Ocasio Del Valle ${ }^{2}$, Jorge A \\ Vargas Venero ${ }^{2}$, and Martty Martínez- Fraticelli ${ }^{3}$ \\ ${ }^{1}$ Department of Pharmaceutical Sciences, Texas A\&M University College of Pharmacy, TX, USA
}

${ }^{2}$ School of Pharmacy, University of Puerto Rico, Puerto Rico

${ }^{3}$ PharmPix Corp, Guaynabo, Puerto Rico

*Corresponding author: José J Hernández Muñoz, Assistant Professor, Department of Pharmaceutical Sciences, Texas A\&M University College of Pharmacy, TX, USA.

To Cite This Article: José J Hernández Muñoz, Prescribers' Use of Electronic Prescribing Inquiry Tools in Puerto Rico. 2020 - 7(4). AJBSR. MS.ID.001163. DOI: 10.34297/AJBSR.2020.07.001163.

Received: 阱 January 27, 2020; Published: 㭗 February 19, 2020

\begin{abstract}
Objectives: To evaluate the use of Electronic Prescribing (E-Prescribing) tools by prescribers generating inquiries to a major Pharmacy Benefit Manager (PBM) in Puerto Rico. A secondary objective is to determine providers' knowledge of E-Prescribing tools.

Methods: This is a retrospective study of E-Prescribing pharmacy claims received by PharmPix. The data collected included the use of E-Prescribing tools, which contain eligibility and medication history verification. The secondary objective was completed with a 15-item questionnaire that collected the prescribers' perception of E-Prescribing systems and tools.

Results: The 10,670 E-Prescribing pharmacy claims generated for the beneficiaries of some groups managed by PharmPix during the first quarter of 2015 were evaluated. Prescribers completed 1,748 (16\%) eligibility requests and $371(3.48 \%)$ medication history verifications for these pharmacy claims. Twenty-two (22) prescribers completed the questionnaire with $77.3 \%$ of prescribers reporting that the E-Prescribing system is easy to use, while $82 \%$ of prescribers agree that the main reason for implementing this software was due to health insurance regulations. More than half of the prescribers know how to use the eligibility status tool in their E-Prescribing software. However, $66.7 \%$ of these prescribers do not use the tool daily. When surveyed about the medical history verification, $81.82 \%$ of prescribers reported successful use of this tool, with $66.67 \%$ prescribers accessing the tool every time they use the software.
\end{abstract}

Conclusion: This study shows the scarce utilization of E-Prescribing tools and the need for future education interventions for prescribers to successfully use the available E-Prescribing systems.

\section{Introduction}

One of the most recent technologies developed in the healthcare setting is Electronic Prescribing, also referred to as E-Prescribing. E-Prescribing is a computer-to-computer transfer of prescription data between pharmacies, prescribers, and payers. The technologies provide results in messages regarding new prescriptions, changes in prescriptions, refill requests, medication history, medication status, and cancellations [1]. All of these characteristics features are managed by Surescripts, an-Electronic Prescribing network, which helps prescribers and pharmacies to improve their standard of care, provide a safe and secure service to patients, and increase their administrative efficiency. Surescripts ensures that E-Prescriptions follow the federal laws that regulate certain specific standards needed for the proper function of the system. Those standards are:

a. Formulary and Benefits (F\&B);

b. Eligibility; and

c. Medication History $(\mathrm{RxH})[2]$.

Most of the benefits obtained from E-Prescribing systems come from the prescriber's access to prescription information. This information is available through the use of uniform standards that enable the E-Prescribing system to exchange information with other data systems. Having the E-Prescription system available 
for prescribers allows them to lower healthcare costs, decrease adverse drug events, increase generic utilization, and have accessibility to formulary information. When E-Prescribing is part of an-Electronic Health Record (EHR), error rates decreased from 42.5 to 6.6 per-100 prescriptions in a year [3]. Access to formulary information helps physicians to prescribe lower-cost medications, leading to estimated savings of $\$ 845,000$ per 100,000 patients [4] The access to this information by the prescriber should shorten the work of pharmacies by sending prescriptions included in the formulary of medical insurance companies, avoiding the need for pre-authorization processes for non-formulary drugs. This type of access is a tool provided by the Pharmacy Benefit Manager (PBM) multiple software vendors, whom are in charge of the communication that exists between prescribers, pharmacies, and the PBMs, and ultimately to insurance companies. Every insurance inquiry received by the PBM indicates the efficient use of the E-Prescribing system by the prescriber as a tool for obtaining the pertinent information of the patient and its insurance coverage. This system promotes the use of cost-effective drugs, resulting in lower healthcare costs.

Having in mind the complete process by which the electronic prescriptions work, it is crucial to evaluate the outcomes and effectiveness of the system in Puerto Rico. Are the prescribers using the insurance inquiry tools of the electronic prescriptions provided by the PBM? Were they aware of the existence of these tools? Are they only using the system as a tool for transcribing computerized prescriptions?

The first objective of this study is to evaluate the use of electronic prescription tools by prescribers generating inquiries to a major PBM in Puerto Rico. The second objective is to determine providers' knowledge on of electronic prescribing tools of prescribers in the PBM database. This study will provide information about physicians in Puerto Rico using the electronic prescription tools, as well as their knowledge regarding this new technology. These results will guide future efforts for training and educating physicians practicing in Puerto Rico about the use and benefits of E-Prescribing.

\section{Material and Methods}

\section{Claim-based Analysis}

The data included in this study will provided by PharmPix through Surescripts and contains the various groups covered by this PBM in three insurance companies. This study will use claims (prescriptions) coming from the PharmPix Electronic Prescribing reservoir for the first quarter (January to March) of 2015. The insurance inquiries submitted by the physicians while writing an electronic prescription will be identified. The identified insurance inquiries will be classified under the following categories: Eligibility, Medication History, and Formulary and Benefits. These claims will be used to measure the amount number of insurance inquiries submitted by the physicians during the first quarter of 2015. This part of the study was conducted retrospectively.

\section{Physicians Interview about E-Prescribing}

After identifying prescribers that sent Electronic prescriptions for the first quarter (January to March) of 2015 for the insurance companies covered by PharmPix, a sample will be selected, and a questionnaire will be administered (Appendix I). The questionnaire will collect information on the prescribers' sociodemographics, type of E-Prescribing software used at their practice and the features they usually use to make insurance inquiries. The questionnaire will be administered face-to-face by the investigators in the physicians' offices to ensure their understanding and honesty when answering the questions of the survey, as well as to witness their use of the Electronic Prescribing software. If any difficulty is encountered which doesn't permit the investigators to visit the physicians' office, the questionnaire will be completed through a previously coordinated telephonic interview. If the physician is not available for a face-to-face or telephonic interview, then the questionnaire will be sent by fax or email as the next option. The physicians will have full communication with the investigators at all times for any questions. This questionnaire does not require validation because it will measure objective data and will not evaluate the physicians' perceptions of E-Prescribing. This part of the project will be conducted concurrently to with the study.

The selected sample size for the interview component will represent $5 \%$ of the doctors working for the covered insurance company groups managed by PharmPix that used the Electronic Prescribing software during the claim based analysis. Due to large quantity number of doctors in the PharmPix database, 60 of the 1,170 physicians will be interviewed as a representative sample of this study. The sample will be selected by simple random sampling, by using a random number generator to match with the prescriber for the face-face or telephonic questionnaire administration. After selecting the prescribers' sample, the investigators will call the medical office to coordinate appointments for the administration of the questionnaire. The estimated time for answering the questionnaire is 15 minutes. If the day of the appointment the doctor is busy and cannot meet with the interviewers, another appointment will need to be scheduled. There will be a maximum of three attempts to coordinate the interview with each selected doctor.
a. Inclusion Criteria
I. Certain Insurance companies groups managed by
PharmPix as a PBM where the contract permits the utilization of data for this purpose
II. Claims from the first quarter (January to March) of 2015
b. Exclusion Criteria 
i. Other insurance companies groups that the contract with PharmPix prevents the use of data for this type of purpose

ii. Claims before January 2015 and after March 2015

Data was analyzed using descriptive statistics as provided by STATA (Version 13) and SAS.

\section{Results}

\section{Part I. Claim-based Analysis}

Overall: For the first quarter (January to March) of 2015, a total of 94,140 prescriptions were obtained from the PharmPix database, of which 10,670 pharmacy claims were part of the E-Prescribing reservoir had the originator code as being an electronic prescription. These claims were distributed by month as follows: 3,460 (32\%) in January, 3,398 (32\%) in February, and 3,812 (36\%) in March. The distribution of the top 50\% E-Prescription claims by pharmacy city were: San Juan 23\%, Bayamón 17\%, Ponce $16 \%$, Carolina 14\%, Caguas 14\%, Humacao 8\%, and Guaynabo 8\%. The distribution of the top $50 \%$ E-Prescription claims by the prescriber city were: San Juan 24\%, Ponce 19\%, Bayamón 16\%, Caguas 13\%, Río Piedras 12\%, Hato Rey 8\%, and Carolina 8\%. Out of the 10,670 E-Prescriptions claims, 4 prescribers did not report the city of origin, for a total of 10,666 claims with city information.

For the 10,670 pharmacy claims, there were a total of 3,051 beneficiaries, 3,046 prescribers, and 645 pharmacies. In an individual analysis of beneficiary city, the top 50\% were: San Juan $27 \%$, Carolina 15\%, Ponce 14\%, Bayamón 14\%, Caguas $10 \%$, Guaynabo 8\%, Humacao 6\%, and Trujillo 6\%.

Eligibility Inquiries: In the first quarter of 2015, the studied groups provided in the PharmPix database contained a total of 94,140 prescriptions. These included 83,470 (89\%) handwritten prescriptions and 10,670 (11\%) E-prescriptions. Patient eligibility was requested for 1,748 E-Prescriptions (16\%). These eligibility requests represent $1.86 \%$ of the total prescriptions analyzed. The eligibility requests were stratified by month (January, February, March). For January, 579 (17\%) eligibility requests of the total 3,460 E-prescriptions for this month. For February, 673 (20\%) eligibility requests of the total 3,398 E-prescriptions for this month. For March, 496 (13\%) eligibility requests of the total 3,812 E-prescriptions for this month. The prescribers inquiring the patient's eligibility for E-prescriptions were 595 (19.53\%) of the 3,046 total prescribers. The distribution of the top $50 \%$ prescribers with the most eligibility inquiries by the prescriber city resulted in San Juan (37.15\%) and Bayamón (16.67\%) at the top of the list. Guaynabo (8.33\%) and Ponce (8.33\%) resulted at in the bottom of the list.

Medication History Verification: Patient medication history verification was requested for 371 E-Prescriptions (3.48\%). These medication history requests represent $0.39 \%$ of the total prescriptions analyzed. The medication history inquiries were stratified by month (January, February, March). For January, 154 (17\%) medication history requests of the total 3,460 E-prescriptions for this month. For February, 119 (20\%) medication history requests of the total 3,398 E-prescriptions for this month. For March, 98 (13\%) medication history requests of the total 3,812 E-prescriptions for this month. The prescribers inquiring the patient's medication history for E-prescriptions were $122(4.01 \%)$ of the 3,046 total prescribers. The distribution of the top $50 \%$ prescribers with the most medication history requests by the prescriber city resulted in San Juan (44.83\%) and Bayamón $(18.97 \%)$ at the top of the list. Caguas $(10.34 \%)$ and Hato Rey $(8.62 \%)$ resulted at in the bottom of the list.

\section{Part II. Prescribers' Questionnaire about E-Prescription Software}

Of the 60 prescribers randomly chosen to complete the questionnaire about E-prescriptions, 53 of them received the questionnaire by fax and 7 of them by e-mail. Out of the 60 questionnaires that were sent (fax/e-mail), 52 of them were confirmed as received by the prescriber. The other 8 questionnaires sent to the prescribers were not confirmed as received because 2 were emergency prescribers without a medical office, 1 prescriber past away recently and 5 prescribers couldn't be contacted. Of the 52 prescribers that received the questionnaire, 22 were completed, 27 were not completed completed, and 3 of them the prescriber denied to complete it. The data of the 22 completed questionnaires were analyzed to obtain information about the prescribers' demographic background and knowledge about the e-prescription program.

\section{Demographics}

Of the 22 prescribers that completed the questionnaire, 16 $(72.7 \%)$ were male and $6(27.3 \%)$ were female. Distribution by the age of the prescribers resulted in $5(22.7 \%)$ between 36 to 45 years, 7 (31.8\%) between 46 to 55 years, 7 (31.8\%) between 56-65 years, and $3(13.6 \%)$ having 65 years or more. Prescribers distribution by medical school where they obtain their medical degree resulted in 12 (54.6\%) from University of Puerto Rico School of Medicine, 1 (4.5\%) from the Ponce School of Medicine, 1 (4.5\%) from the San Juan Bautista School of Medicine, and 8 (36.4\%) from other medical schools. Distribution by prescribers by medical specialty resulted in 4 (18\%) internist, 3 (14\%) gastroenterologist, 2 (9\%) obstetriciangynecologists, 3 (14\%) generalists, 1 (4\%) cardiologist, 1 (4\%) psychiatrist, $1(5 \%)$ pediatrician, and 7 (32\%) with other specific medical specialty. Prescribers' years of medical practice obtained from the questionnaire were $5(22.7 \%)$ between 8 to 14 years of practice, $5(22.7 \%)$ between 15 to 21 years of practice, $4(18.2 \%)$ between 22 to 28 years of practice, 7 (31.8\%) between 29 to 35 years of practice, and 1 (4.6\%) having 36 years or more of medical practice. 


\section{Perceptions about E-Prescribing Program}

Prescribers reported the use of the E-Prescribing software for 5 years $(7 ; 31.8 \%)$, 4 years $(5 ; 22.7 \%), 3$ years $(4 ; 18.2 \%)$, and 2 years (5; 22.7\%). The E-Prescribing software mostly used by prescribers include Practice Fusion (5; 22.6\%), Neo-Med (4;18.2\%), EHRe2 (4;18.2\%), and Inmediata (4;18.2\%). Training for accessing the software was completed by $77 \%$ of the prescribers. The training received included personalized training with a technician by 12 prescribers and interactive training with the software program by 6 prescribers. The prescribers that did not perceive the E-Prescribing software to have a high difficulty level were 17 prescribers $(77.3 \%)$. The reasons reported for implementing the use of E-prescriptions in their medical offices included: minimize ilegible letter in medical prescriptions (10 prescribers), a easier more straightforward delivery method of sending a prescription to the pharmacy (8 prescribers), and due to prescribing laws (18 prescribers). Some prescribers complete only an electronic delivery of the prescription to the pharmacy $(11 ; 50 \%)$, while others send the E-Prescription electronically and also print a hardcopy for the patient (10; 45.6\%). Most prescribers prefer the writing an electronic prescription (17; $77.3 \%$ ) over a handwritten prescription $(5 ; 22.7 \%)$.

More than half of the prescribers $(12 ; 54.55 \%)$ reported that their E-Prescribing software contained the option to access patient's eligibility status. Only 1 prescriber (4.55\%) reported not having access to verify eligibility status, while 8 prescribers (36.36\%) had no knowledge of the tool. Out of the 12 prescribers that reported having access to the patient's eligibility status, 2 $(16.67 \%)$ accessed the tool every time they used the software, $8(66.67 \%)$ did not use the tool, and $2(16.67 \%)$ used the tool sometimes. When asked if their E-Prescribing software contained the option to access the patient's medication history, 18 prescribers (81.82\%) reported positively, 1 prescriber (4.55\%) reported negatively, and 3 prescribers (13.64\%) had no knowledge of the tool. Out of the 18 prescribers that reported having access to the patient's medication history, $12(66.67 \%)$ accessed the tool every time they used the software, $1(5.56 \%)$ did not use the tool, and $5(27.78 \%)$ used the tool sometimes. Half of the prescribers (11; $50 \%$ ) reported that they had no knowledge that their E-prescribing software contained the option to access formulary and benefits. Only 4 prescribers $(18.18 \%)$ reported having access to formulary and benefits, while 7 prescribers $(31.82 \%)$ reported not having access to the tool. Out of the 4 prescribers that reported being able to access the patient's formulary and benefits, 1 (25\%) accessed the tool every time he used the software, while $3(75 \%)$ did not use the tool. Most prescribers $(14 ; 63.64 \%)$ recognize or have heard about Surescripts, while 8 prescribers (36.36\%) have no knowledge.

\section{Discussion}

The implementation of E-Prescribing softwares at the prescribers' office in Puerto Rico started six years ago. However, this relatively new technology has not been evaluated to determine if it is currently meeting the goals that contributed to its creation. Some of these goals are increasing the quality of care provided to patients and the cost-effectiveness of drug therapy, while reducing prescribing errors. $(3,4)$. In the first quarter of 2015 , the studied groups provided in the PharmPix database contained a total of 94,140 prescriptions. These included 83,470 (89\%) handwritten prescriptions and only 10,670 (11\%) E-prescriptions.

The data analyzed in Part I of the study showed the prescribers' lack of use of the eligibility and medication history verification tools included as standards for E-Prescribing systems [1,2]. From the 10,670 E-Prescription pharmacy claims contained in the PharmPix database for the first quarter of 2015, prescribers completed 1,748 (16\%) eligibility requests and 371 (3.48\%) medication history inquiries. This data shows that prescribers are not using the tools for which E-Prescribing software implementation is advocated. This could be due to a lack of existence of these tools in their acquired software, no interest in investing time in these systems or insufficient education on how to correctly use the E-Prescribing tools.

In Part II of the study, the prescribers reported the availability of numerous softwares for generating E-Prescriptions. This may suggest that E-Prescribing systems could differ in their design and difficulty level influencing prescriber use, as reported by Grossman et al. [5]. Although $77 \%$ of prescribers received training on their E-Prescribing software, continued emphasis on education will prove to be beneficial by contributing to better use of the system tools [5]. On the basis of based on difficulty level in our study, $77.3 \%$ of prescribers reported that the E-Prescribing system is easy to use. In a similar study by Wang et al. 79\% of E-Prescribers reported that the system was easy to use as well [6]. Additionally, prescribers agree that the main reason for implementing E-Prescriptions software in their medical office was due to health insurance regulations.

More than half of the prescribers' know how to use the eligibility status tool in their E-Prescribing software. However, $66.7 \%$ of these prescribers do not use the tool on a daily basis. When surveyed about the medical history, most prescribers (81.82\%) reported successful access to this tool. A similar survey to physicians discovered that $62 \%$ had access to medication histories and, of these physicians, $56 \%$ used this tool most or all of the time [7].

In our study, few prescribers $(18.18 \%)$ have access to the formulary and benefits tool and, of these prescribers, only 1 (25\%) accessed the tool every time he used the software. This finding shows the lack of knowledge or availability of this tool in the E-Prescribing systems. A survey to physicians in a study by Desroches et al. reported that $60 \%$ had access to formulary information and, of these physicians, $34 \%$ used this tool most or all of the time [7]. 
This study has several limitations. First, Part I of this study contains the data of a single PBM, it is not inclusive of all available insurance companies nor of all prescribers' practices within their E-Prescribing software. It represents a sample from the first quarter of 2015, covering data from just three months. Second, Part II of this study contains a small sample size, self-administered questionnaire, the variety of available E-Prescribing systems, and the difficulty to reaching randomly selected prescribers for our study.

\section{Conclusion}

This is the first study analyzing the use of E-Prescribing tools in Puerto Rico and the prescribers' perception of this electronic system. The E-Prescription system primary purpose is to provide prescribers an improvement in the quality and safety of the prescribing process. Nevertheless, claims from the PBM PharmPix shows an inappropriate use of the system tools. The scarce utilization of E-Prescribing tools and the need of for future education interventions for prescribers are important essential factors that need to be improved for the appropriate use of the E-Prescribing system in Puerto Rico.

\section{References}

1. (2008) A Clinician's Guide to Electronic Prescribing. Washington, US. The Center for Improving Medication Management p. 1-38.

2. Levinson DR (2009) Medicare Part D E-Prescribing Standards: Early assessment shows partial connectivity. Department of Health and Human Services.

3. Porterfield A, Engelbert K, Coustasse A (2014) Electronic prescribing: improving the efficiency and accuracy of prescribing in the ambulatory care setting. Perspect Health Inf Manag 11(1): 1g.

4. Fischer MA, Vogeli C, Stedman M, Ferris T, Brookhart MA, et al. (2008) Effect of electronic prescribing with formulary decision support on medication use and cost. Arch Intern Med 168(32): 2433-2439.

5. Grossman JM, Boukus ER, Cross DA, Cohen GR (2011) Physician practices, e-prescribing, and accessing information to improve prescribing decisions. Res Brief (20): 1-10.

6. Wang CJ, Patel MH, Schueth AJ, Glassman PA, Bell DS, et al. (2009) Perceptions of standards-based electronic prescribing systems as implemented in outpatient primary care: a physician survey. J Am Med Inform Assoc 16(4): 493-502.

7. DesRoches, Catherine M, Agrawal R, Fischer MA (2010) Differences between Integrated and Stand-Alone E-Prescribing Systems Have Implications for Future Use. Health Affairs 29(12): 2268-2277 